\title{
Signal Frequency Estimation Based on Kalman Filtering Method
}

\author{
Yandu LIU, Di YAN and Haixin ZHENG \\ Equipment Academy, 101416 Beijing, China
}

\begin{abstract}
In order to further improve the frequency estimation precision under high dynamic, the frequency estimation method based on Kalman filter is studied. Two methods of Kalman filter directly using for frequency estimation are studied, taking the phase of echo signal and the echo signal for measurements, which adopt nonlinear and linear Kalman filtering algorithm to estimate the Doppler frequency. Based on the study of performance of these two methods, the Doppler adaptability of the two methods is studied. The simulation results show that the speed precision is improved. But, the two methods of this research are limited by the fixed frequency conversion, and when Doppler changed a large range, the filtering is easy to diverge.
\end{abstract}

\section{Introduction}

LEO satellite flight dynamics complicated, speed and all derivative values are large, so it belongs to frequency estimation problem under high dynamic condition. Phaselocked loop is the most commonly used in satellite signal processing frequency estimation method[1-4], but under high dynamic conditions it requires using higher-order loop, which cause stability problems. Kalman filter is an optimal estimation method, with excellent performance and noise filtering characteristics predict $[5,6]$. In order to further improve the high frequency dynamic state estimation accuracy, this paper focus on the frequency estimation method based on Kalman Filter.

\subsection{Kalman Filter}

Kalman filter is a recursive process, the current estimate of the time and past time-related and affects an estimated value of future time. Discrete linear system model is [7]:

$$
\begin{gathered}
\mathbf{X}_{k}=\boldsymbol{\Phi}_{k, k-1} \mathbf{X}_{k-1}+\boldsymbol{\Gamma}_{k, k-1} \mathbf{W}_{k} \\
\mathbf{Z}_{k}=\mathbf{H}_{k} \mathbf{X}_{k}+\mathbf{V}_{k}
\end{gathered}
$$

$\boldsymbol{\Phi}_{k, k-1}$ is a state deviation transition matrix, $\mathbf{H}_{k}$ is measurement matrix, which represents the relationship between the amount of measurement and status. $\Gamma_{k, k-1}$ is System noise driving matrix, and the statistical characteristics of the system noise $\mathbf{W}_{\mathrm{k}}$ and measurement noise $\mathbf{V}_{k}$ is:

$$
\begin{gathered}
E\left[\mathbf{W}_{k}\right]=0, \mathbf{R}_{\mathrm{WW}}(k, j)=\mathbf{Q}_{k} \delta_{k j} \\
E\left[\mathbf{V}_{k}\right]=0, \mathbf{R}_{\mathrm{VV}}(k, j)=\mathbf{R}_{k} \delta_{k j} \\
\mathbf{R}_{\mathrm{WV}}(k, j)=0
\end{gathered}
$$

The statistical characteristics of initial state $\mathbf{X}_{0}$ is :

$E\left[\mathbf{X}_{0}\right]=\overline{\mathbf{X}}_{0}, \operatorname{Var}\left[\mathbf{X}_{0}\right]=\mathbf{P}_{0}$

So the basic process of Kalman filter is as follows:

Step 1: Calculation step prediction of mean square error matrix.

$$
\mathbf{P}_{k, k-1}=\mathbf{\Phi}_{k, k-1} \mathbf{P}_{k-1} \boldsymbol{\Phi}_{k, k-1}^{T}+\boldsymbol{\Gamma}_{k, k-1} \mathbf{Q}_{k-1} \boldsymbol{\Gamma}_{k, k-1}^{T}
$$

Step 2: Calculation state step prediction.

$$
\hat{\mathbf{X}}_{k, k-1}=\boldsymbol{\Phi}_{k, k-1} \mathbf{X}_{k-1}
$$

Step3: Calculation Kalman filter gain.

$$
\mathbf{K}_{k}=\mathbf{P}_{k, k-1} \mathbf{H}_{k}^{T}\left(\mathbf{H}_{k} \mathbf{P}_{k, k-1} \mathbf{H}_{k}^{T}+\mathbf{R}_{k}\right)^{-1}
$$

Step4: Calculation mean square error matrix of filter.

$$
\mathbf{P}_{k}=\left(\mathbf{I}-\mathbf{K}_{k} \mathbf{H}_{k}\right) \mathbf{P}_{k, k-1}
$$

Step5: Calculation Status filter value.

$$
\mathbf{X}_{k}=\mathbf{\Phi}_{k, k-1} \mathbf{X}_{k-1}+\mathbf{K}_{k}\left[\mathbf{r}_{k}-\mathbf{h}\left(\hat{\mathbf{X}}_{k, k-1}\right)\right]
$$

Step 1 and step 2 push the time from k-1 moment to $\mathrm{k}$ moment, so it is called time update process; step 3,4,5 using the observed quantity on time to update the value, so it is called measurement update process.

\subsection{Phase Locked LOOP(PLL) and Kalman FILter(KF)}

Kalman filter's time update equation and measurement update equations are:

$$
\begin{gathered}
\hat{\mathbf{X}}_{k+1, k}=\boldsymbol{\Phi}_{k+1, k} \mathbf{X}_{k} \\
\mathbf{X}_{k}=\hat{\mathbf{X}}_{k, k-1}+\mathbf{K}_{k}\left[\mathbf{y}_{k}-h\left(\hat{\mathbf{X}}_{k, k-1}\right)\right]
\end{gathered}
$$

Combined type two, then:

$$
\hat{\mathbf{X}}_{k+1, k}=\boldsymbol{\Phi}_{k+1, k} \hat{\mathbf{X}}_{k, k-1}+\boldsymbol{\Phi}_{k+1, k} \mathbf{K}_{k}\left[\mathbf{y}_{k}-h\left(\hat{\mathbf{X}}_{k, k-1}\right)\right]
$$

In order to facilitate the derivation of transfer function, neglect the system noise and the measurement noise matrix. Fig. 1 is the recursive process of the formula (14). 


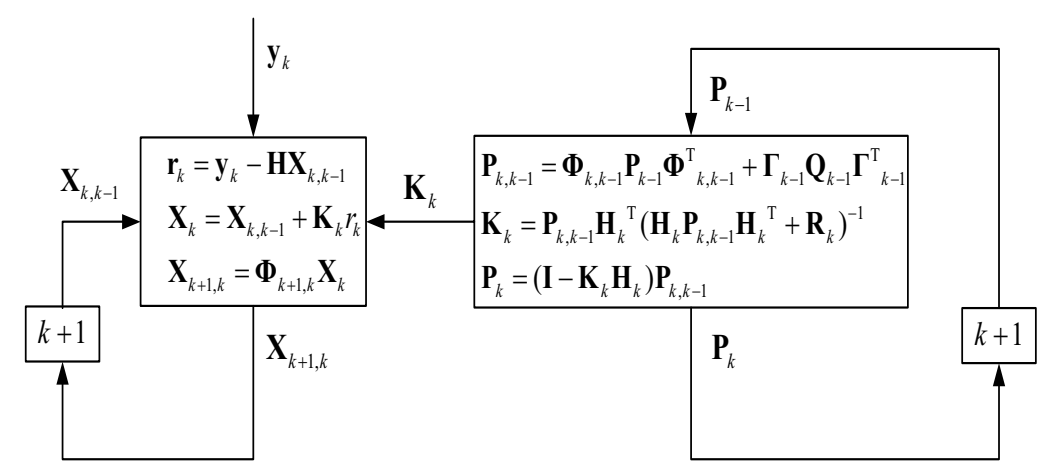

Fig. 1. Kalman filtering recursive process.

The recursive process is converted into the structure diagram of the algorithm shown in Fig.2, and the delay module $z^{-1}$ in the loop is the estimate of the current time prediction, and the module $\mathrm{k}+1$ in Fig. 1 is corresponding to the step estimate $[8,9]$.

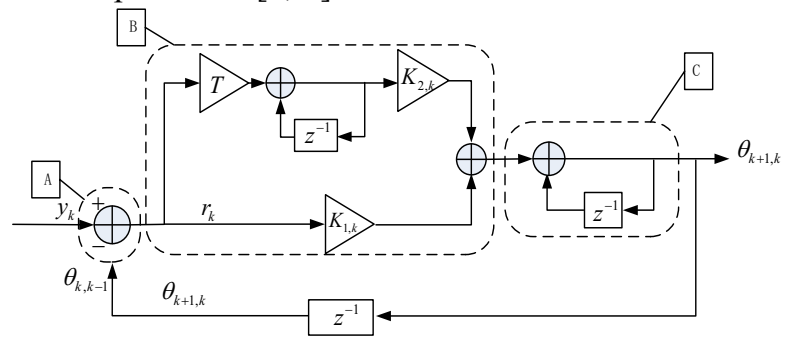

Fig. 2. Kalman filtering structure.

The overall structure of the Fig. 2 and PLL is very similar, compared with the transfer function of PLL and KF, Part $\mathrm{A}$ is equivalent to the phase detector, Part $\mathrm{B}$ is in similar to the PLL loop filter and Part $\mathrm{C}$ is equivalent to the $\mathrm{NCO}$ in PLL.

\section{Kalman frequency measurement}

Through the study of the last section, the estimation accuracy of the Kalman filter and the SNR threshold are better than the PLL. In this section, the method of estimating the frequency of Kalman filter is studied directly. According to the difference of measurement, it is divided into two methods. First method is a EKF filter without phase detection, and the measurement is the signal itself. Second method is a KF filtering with the phase detector, and measurement is the phase of the phase detector output.

\subsection{EKF method without phase detector}

Principle block diagram as shown in Fig. 3, intermediate frequency (IF) signal is mixed and shifted to the baseband. Then feed into the Kalman filter after resample. Because of the nonlinear relationship between the measurement and the phase, EKF algorithm is used.

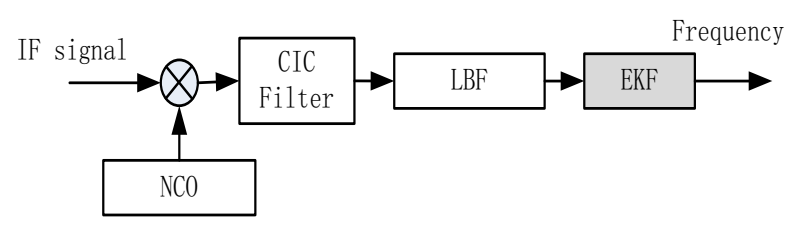

Fig. 3. EKF filter without phase detection method.

The CIC extractor and the low-pass filter generate different amplitude gain for different frequency offset signal, and the difference is large. So the amplitude of the state vector is increased, and the five order EKF is formed.

State vector is:

$$
\mathbf{X}=\left[\begin{array}{lllll}
A & \theta & \Delta \omega_{0} & \Delta \omega_{1} & \Delta \omega_{2}
\end{array}\right]^{T}
$$

$A$ is signal amplitude, $\theta$ is the phase, and the status transfer matrix is[10]:

$$
\boldsymbol{\Phi}_{k, k-1}=\left[\begin{array}{ccccc}
1 & 0 & 0 & 0 & 0 \\
0 & 1 & 2 \pi T & \pi T^{2} & \frac{1}{3} \pi T^{3} \\
0 & 0 & 1 & 2 \pi T & \pi T^{2} \\
0 & 0 & 0 & 1 & 2 \pi T \\
0 & 0 & 0 & 0 & 1
\end{array}\right]
$$

And the Measurement matrix is:

$$
\mathbf{H}=[\sin (x(2)) \quad x(1) \times \cos (x(2)) \quad 0 \quad 0 \quad 0]
$$

In order to validate the algorithm, the signal of $70 \mathrm{dBHz}$ and $50 \mathrm{dBHz}$ is simulated, as shown in Fig.4. The results are compared with the theoretical values. The simulation results show that the new five order EKF model is correct. The theoretical amplitude $A_{\text {theory }}$ is calculated as (18) [11]

$$
A_{\text {theory }}=\sqrt{\frac{\sum()^{2} / N}{1+\frac{1}{10^{S N R / 10}}}}
$$

$\mathrm{N}$ is the signal points, SNR is the known SNR. The comparison between the amplitude and the theoretical value is shown in Fig.4 and Fig.5. 


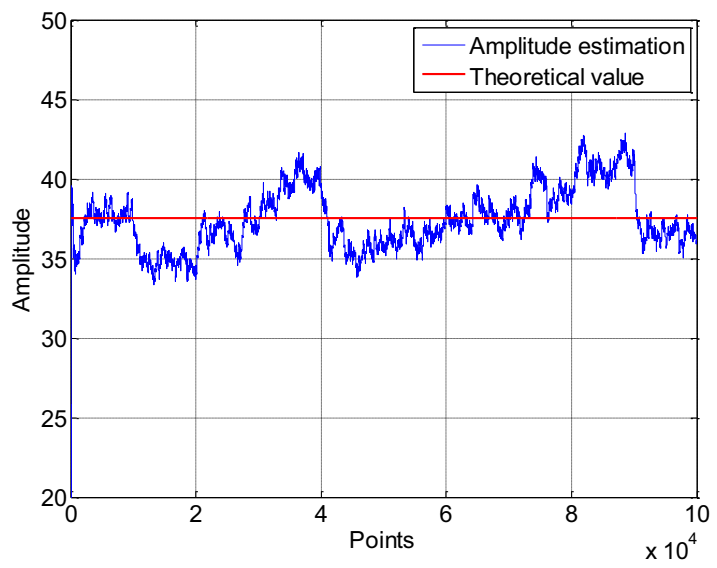

Fig. 4. $\mathrm{SNR}=50 \mathrm{dBHz}$

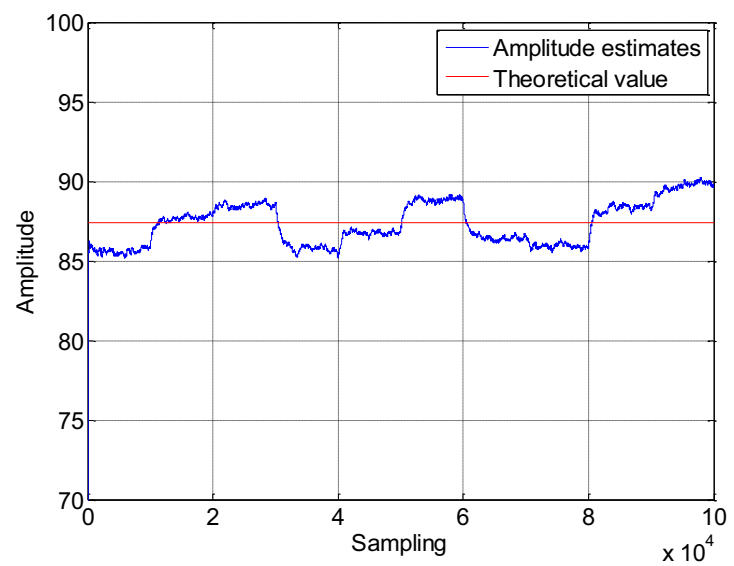

Fig. 5 . $\mathrm{SNR}=70 \mathrm{dBHz}$.

\subsection{KF method with phase detector}

In the last section, using EKF algorithm, the nonlinear error is introduced in the process of model linearization, and the total estimation error of the nonlinear error in high SNR can be large. In order to avoid nonlinear error introduced by the linearization of the nonlinear model, with the linear variation of the phase measurements, using a linear KF algorithm, as shown in Fig.6, with the aid of phase detecting is studied.

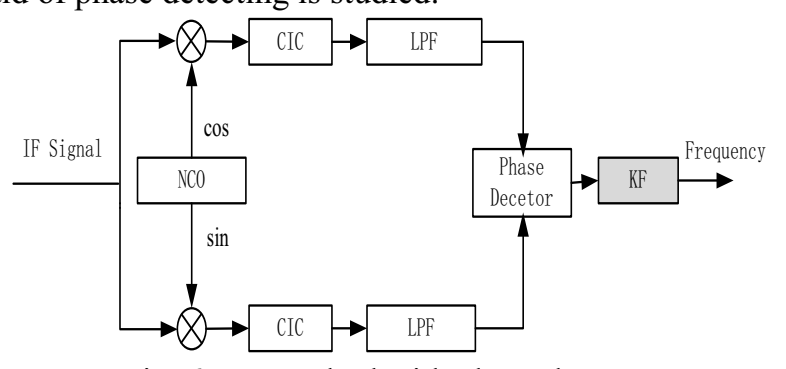

Fig. 6. KF method with phase detector

Actual signal phase is continuously with time increasing, but the arctangent discriminator phase shifting to range $\left[-\frac{\pi}{2}, \frac{\pi}{2}\right]$, the phase value is periodically from $\frac{\pi}{2}$ mutations to $-\frac{\pi}{2}$, resulting in new information changes caused by the estimated value of the shock. Using the method of identifying the phase mutation and improving the new information, the method can effectively inhibit the effect of the phase mutation.

The comparison of the results of the new interest calculation method before and after the improvement of the new information is made by simulation.

The method shown in Fig.7 can better solve the filtering instability caused by the measurement of the amount of the measurement, and not only recover the carrier phase accurately and estimate the residual Doppler frequency accurately.

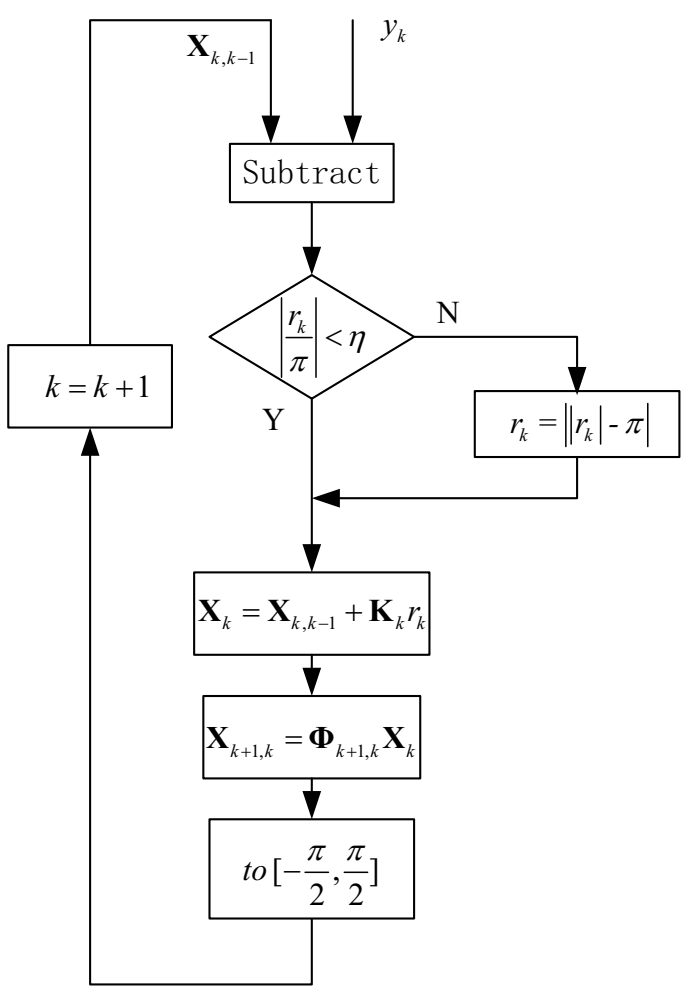

Fig. 7. Improvement of the new interest calculation method process.

When $S N R=45 \mathrm{dBHz}, f_{\mathrm{d}}=20 \mathrm{~Hz}$, The results of the phase and frequency estimation are shown in Fig. 8.

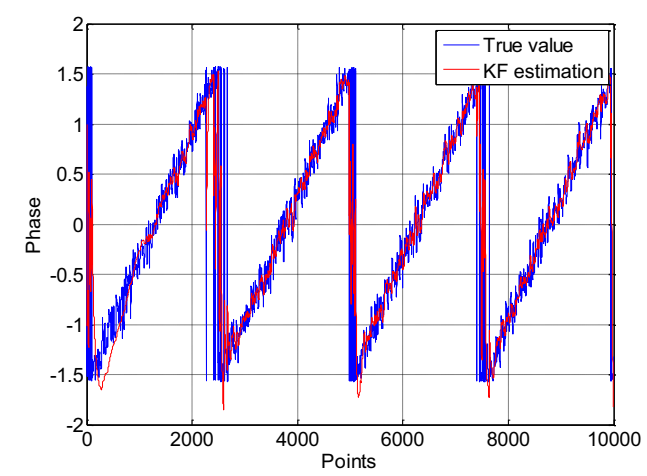

Fig.8(a). Former phase estimator compared with true value before the improvement 


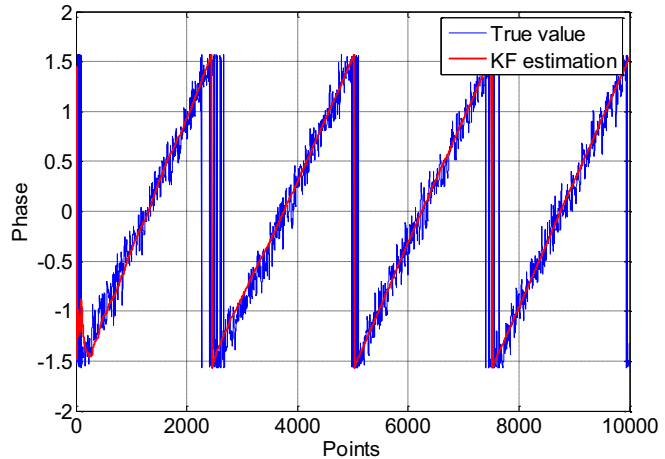

Fig. 8(b). Improved phase estimator compared with true value

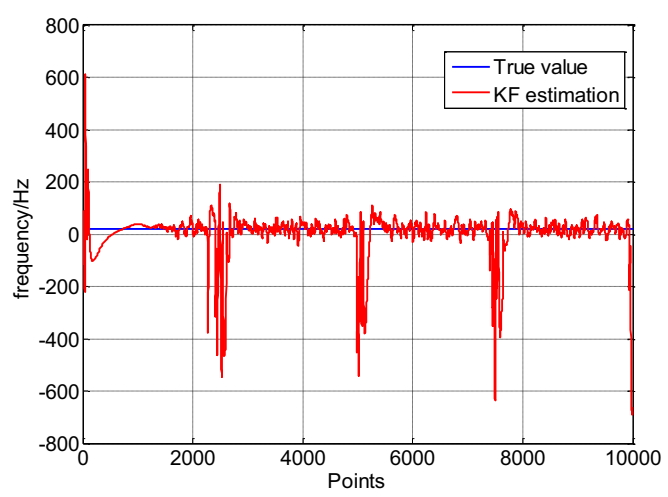

Fig.8(c). Former frequency estimator compared with true value before the improvement

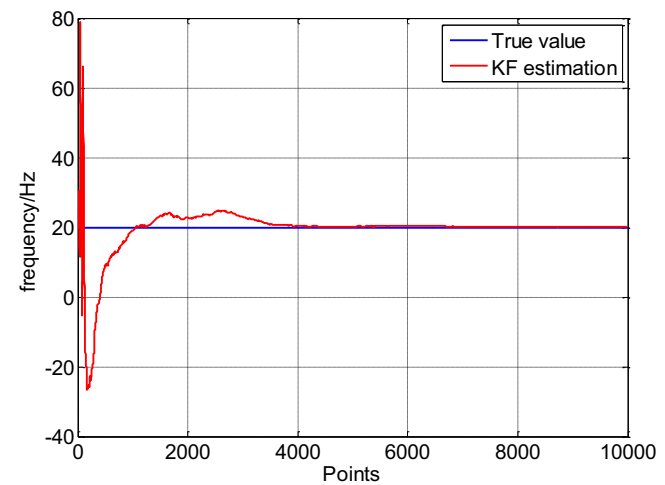

Fig.8(d). Improved frequency estimator compared with true value

Fig. 8 shows that point mutations in the phase, improved phase estimation results in the presence of multiple zero crossing frequency estimation results big mutation; the results of improved phase estimation is more smooth, and no mutation in frequency estimation results.

When $S N R=45 \mathrm{dBHz}, f_{d}=500+1000 t \mathrm{~Hz}$, The results of the phase and frequency estimation are shown in Fig.9.

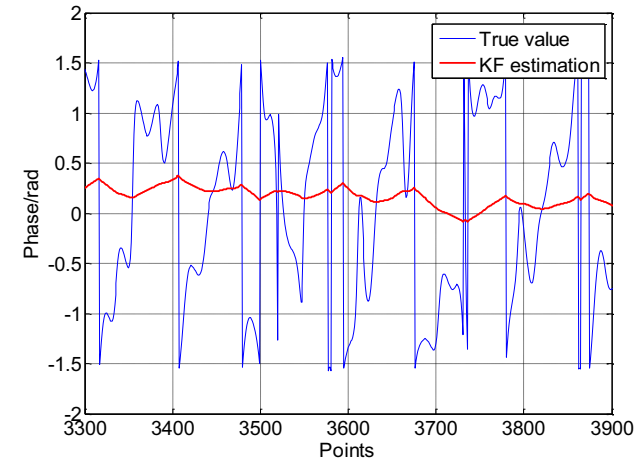

Fig.9(a). Former phase estimator compared with true value before the improvement

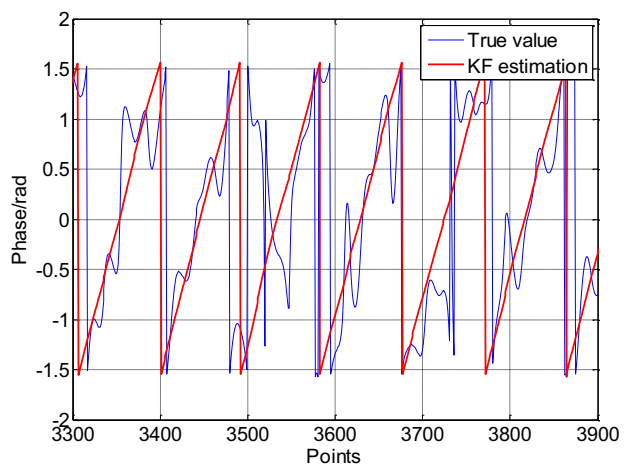

Fig.9(b). Improved phase estimator compared with true value

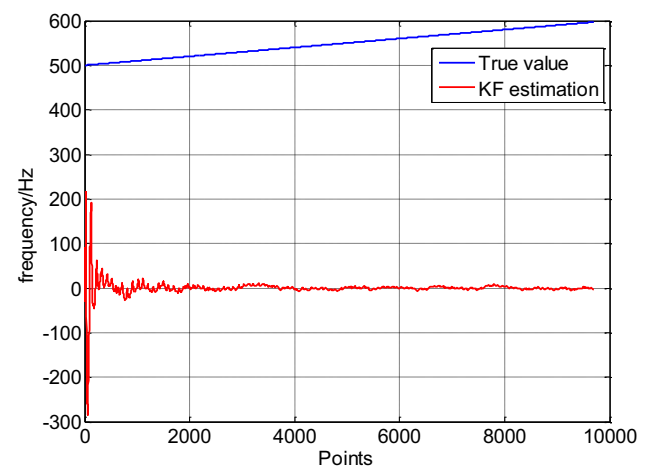

Fig.9(c). Former frequency estimator compared with true value before the improvement

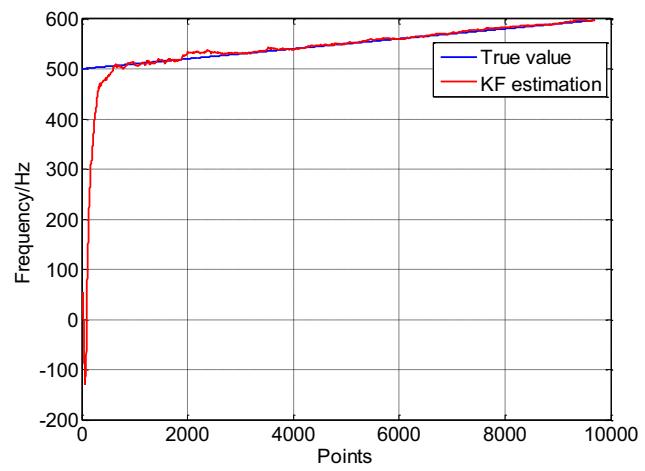

Fig.9(d). Improved frequency estimator compared with true value

Fig.9 shows that the Kalman filter appeared divergent phenomenon when the Doppler frequency is large, and 
the improved Kalman filter can recover the phase and frequency information accurately.

\section{Frequency estimation correction}

The two method is pre - processing of FIR filter before the processing of Kalman filter, which brings the time delay of fixed point number. Set the order of filters $M=50$, signal doppler first rate of change is $\dot{f}_{\mathrm{d}}=10000 \mathrm{~Hz} / \mathrm{s}$, doppler error caused by delay is $\Delta f_{\mathrm{d}}$,so $\Delta f_{\mathrm{d}}=\frac{M}{2} T_{\mathrm{S}} \dot{f}_{\mathrm{d}}=2.5 \mathrm{~Hz}$

$T_{\mathrm{S}}$ is the signal sampling period, $\frac{M}{2} T_{\mathrm{S}}$ is the delay of the filter. As shown in Fig.10, the Doppler error caused by the delay.

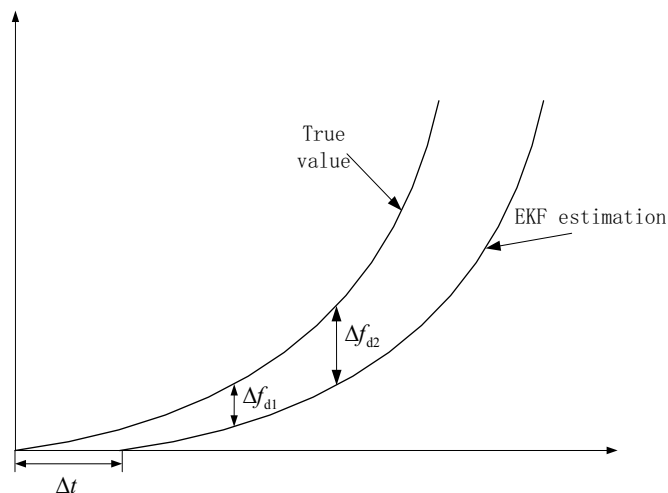

Fig.10. Improvement of the new interest calculation method process.

Fig.10 shows that with the simulation time, the Doppler error caused by the delay is becoming bigger and bigger. The error of the delay must be corrected. Let the state vector $\mathbf{X}_{k}$ be obtained after setting the moment $\mathrm{k}$, and the modified state quantity $\mathbf{X}_{k c o r r}$ is

$$
\mathbf{X}_{k c o r r}=\left[\begin{array}{ccccc}
1 & 0 & 0 & 0 & 0 \\
0 & 1 & 2 \pi T & \pi T^{2} & \frac{1}{3} \pi T^{3} \\
0 & 0 & 1 & T & \frac{1}{2} T^{2} \\
0 & 0 & 0 & 1 & T \\
0 & 0 & 0 & 0 & 1
\end{array}\right] \mathbf{X}_{k}
$$

$T=\frac{M}{2} T_{\mathrm{S}}$ is the delay of the filter is introduced.

\section{Frequency Estimation performanc analysis}

There are two main error sources of EFK method, error $\sigma_{11}$ caused by measurement error and $\sigma_{12}$ caused by linearization of the EFK. FK Method estimates of the error source is only one: the error due to measurement noise $\sigma_{2}$.

Set input intermediate frequency signal is:

$$
s(t)=\sin \left[2 \pi\left(f_{\mathrm{c}}+f_{\mathrm{d}}\right) t+\varphi\right]+n(t)
$$

$f_{\mathrm{c}}$ is signal frequency, $f_{\mathrm{d}}$ is Doppler frequency, $\varphi$ is the early phase, $n(t)$ is white noise sequence and $E\left(n^{2}\right)=\sigma^{2}$.

$\mathrm{NCO}$ output two orthogonal signals are:

$$
\begin{aligned}
N_{\mathrm{I}}(t) & =\sin \left(2 \pi f_{\mathrm{c}} t\right) \\
N_{\mathrm{Q}^{\mathrm{Q}}}(t) & =\cos \left(2 \pi f_{\mathrm{c}} t\right)
\end{aligned}
$$

After mixing and filter the double frequency:

$$
\begin{gathered}
I(t)=A \sin \left(2 \pi f_{\mathrm{d}} t+\varphi\right)+n_{\mathrm{I}}(t) \\
Q(t)=A \cos \left(2 \pi f_{\mathrm{d}} t+\varphi\right)+n_{\mathrm{Q}}(t)
\end{gathered}
$$

The size of $\mathrm{A}$ is caused by CIC extraction filter gain

$$
E\left(n_{\mathrm{I}}^{2}\right)=E\left(n_{\mathrm{Q}^{2}}\right)=A^{2} \sigma^{2}
$$

After the arctangent :

$$
y(t)=\tan ^{-1}\left(\frac{I(t)}{Q(t)}\right)
$$

the noise sequencey translated by $I(t), Q(t)$ to $y(t)$ is $n_{\mathrm{y}}(t)$, the variance is $\sigma_{\mathrm{y}}^{2}$, when:

$$
y(t)=2 \pi f_{\mathrm{d}} t+\varphi+n_{\mathrm{y}}(t)
$$

According to the principle of error transfer [11]

$$
\begin{aligned}
\sigma_{\mathrm{y}}^{2} & =\left(\frac{\partial y}{\partial I}\right)^{2} \sigma_{\mathrm{I}}^{2}+\left(\frac{\partial y}{\partial Q}\right)^{2} \sigma_{\mathrm{Q}}^{2} \\
& =\left(\frac{Q}{I^{2}+Q^{2}}\right)^{2} A^{2} \sigma^{2}+\left(\frac{I}{I^{2}+Q^{2}}\right)^{2} A^{2} \sigma^{2} \\
& =\frac{A^{2} \sigma^{2}}{I^{2}+Q^{2}} \\
& =\sigma^{2}
\end{aligned}
$$

To Solving $\frac{\partial y}{\partial I} 、 \frac{\partial y}{\partial Q}$ theoretical expression of $I(t), Q(t)$ is used when:

$$
\begin{aligned}
& I(t)=A \sin \left(2 \pi f_{\mathrm{d}} t+\varphi\right) \\
& Q(t)=A \cos \left(2 \pi f_{\mathrm{d}} t+\varphi\right)
\end{aligned}
$$

EKF method use $I(t)$ as measurement, and KF method use $y(t)$ as measurement. In order to facilitate the input of the two methods, $I(t)$ will be normalized as:

$$
\begin{gathered}
I^{\prime}(t)=\sin \left(2 \pi f_{\mathrm{d}} t+\varphi\right)+n^{\prime}(t) \\
E\left(n^{\prime 2}\right)=\sigma^{2}
\end{gathered}
$$

Type(30) and type(27) of noise power are the same, but the signal form. The measurement in type(27) is the linear function of state vector, but the measurement in type(30) is the nonlinear function of state vector, which makes the type(27) can use KF algorithm. Meanwhile the type(30) can only use EKF algorithm which just introduce nonlinear error $\sigma_{12}$. Only measurement matrix calculation methods of EKF and KF algorithms are different, so the main recursive process almost unanimously, also using the same parameters in practical application, so the estimated error caused by the 
measurement noise is the same. Therefore, under normal circumstances, the KF method is better than the EKF method, when $\sigma_{11}+\sigma_{12}>\sigma_{2}$.

Compared the accuracy of the estimation of the two methods, the simulation results are shown in Fig. 11.

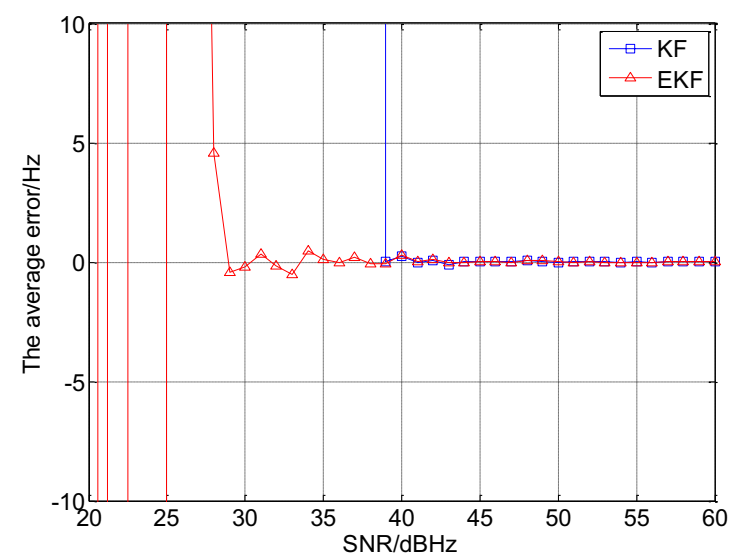

Fig.11(a). Average error of frequency estimation under different SNR.

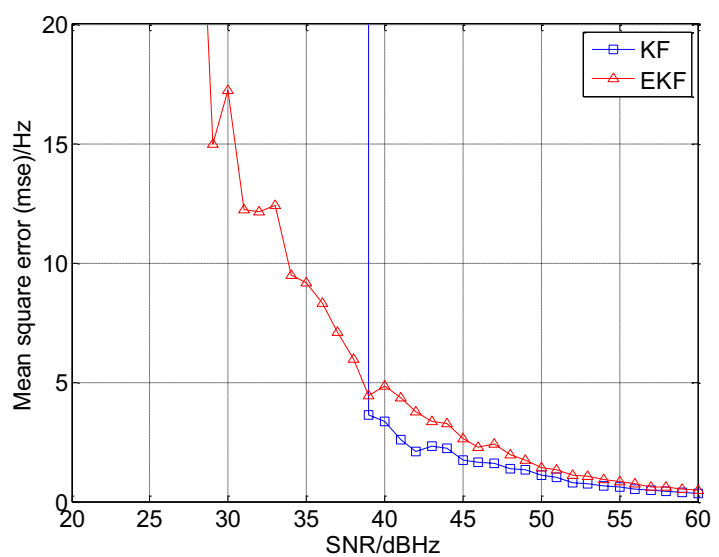

Fig.11(b). The root mean square error of frequency estimation under different SNR

\section{Conclusions}

Fig. 11 shows that the EKF method of a SNR threshold is lower than that of the KF method, which is because the periodic mutation of the measurement has more influence on the algorithm under the low SNR, resulting in the divergence of the algorithm. However, when the SNR threshold is above, KF method, the accuracy is higher than that of the EKF method.

Therefore, the two methods of this research are limited by the fixed frequency conversion, and when Doppler changed a large range, the filtering is easy to diverge. If the closed-loop structure of PLL is introduced, the residual Doppler frequency after the frequency conversion is maintained at the appropriate interval, and the above divergence can be avoided.
1. Wagdy M F, Vaishnava S. A Fast-Locking Digital Phase-Locked Loop[C]//Proceedings of the Third International Conference on Information Technology: New Generations. IEEE Computer Society, 2006: 742-746.

2. El-Laithy D M, Zekry A, Abouelatta M. Speedingup Phase-Locked Loops based on Adaptive Loop Bandwidth [J]. International Journal of Computer Applications, 2013, 61(3): 1-6.

3. Rao M, Lo Presti L, Fantino M, et al. A software receiver adaptive phase lock loop method [J]. 2009.

4. Aung M, Hurd W J, Buu C M, et al. The Block V Receiver fast acquisition algorithm for the Galileo S-band mission [J]. 1994.

5. Vilnrotter V A, Hinedi S, Kumar R. Frequency estimation techniques for high dynamic trajectories[J]. Aerospace and Electronic Systems, IEEE Transactions on, 1989, 25(4): 559-577.

6. Natali F D. AFC tracking algorithms [J]. Communications, IEEE Transactions on, 1984, 32(8): 935-947.

7. Anguirre S, Hinedi S. Two novel automatic frequency tracking loops $[\mathrm{J}]$. Aerospace and Electronic Systems, IEEE Transactions on, 1989, 25(5): 749-760.

8. Hurd W J, Statman J I, Vilnrotter V A. High dynamic GPS receiver using maximum likelihood estimationand frequency tracking $[\mathrm{J}]$. Aerospace and Electronic Systems, IEEE Transactions on, 1987 (4): 425-437.

9. Aguirre S. An automatic frequency control loop using overlapping DFTs [J]. Telecommunications and Data Acquisition Progress Report, 1988, 94: 222-231.

10. Hinedi S. An extended Kalman filter based automatic frequency control loop[C]/The Telecommunications and Data Acquisition Report. 1988, 1: 219-228.

11. Wang J, Liang Q, Liang K, et al. A new extended Kalman filter based carrier tracking loop[C]//2009 3rd IEEE International Symposium on Microwave, Antenna, Propagation and EMC Technologies for Wireless Communications. 2009: 1181-1184.

\section{References}

